\title{
Correlates of whole-blood polyunsaturated fatty acids among young children with moderate acute malnutrition
}

\author{
C. W. Yaméogo 1,2,3* B. Cichon², C. Fabiansen2, M. J. H. Rytter², D. Faurholt-Jepsen²,4 K. D. Stark ${ }^{5}$, A. Briend ${ }^{2,6}$, \\ S. Shepherd ${ }^{7}$, A. S. Traoré ${ }^{1}$, V. B. Christensen ${ }^{2,8}$, K. F. Michaelsen ${ }^{2}$, H. Friis ${ }^{2}$ and L. Lauritzen ${ }^{2}$
}

\begin{abstract}
Background: Severe acute malnutrition (SAM) has been associated with low polyunsaturated fatty acid (PUFA) status. However, investigations regarding PUFA status and correlates in children with moderate acute malnutrition (MAM) from low-income countries are scarce. The aim of this study was to describe whole-blood PUFA levels in children with moderate acute malnutrition (MAM) and to identify correlates of PUFAs.
\end{abstract}

Methods: We conducted a cross-sectional study using baseline data from a prospective nutritional intervention trial among 1609 children with MAM aged 6-23 months in Burkina Faso,West Africa. Whole-blood PUFAs were measured by gas chromatography and expressed as percent of total whole-blood fatty acids (FA\%). Potential correlates of PUFAs including infection, inflammation, hemoglobin, anthropometry (difference between children diagnosed as having MAM based on low mid-upper-arm-circumference (MUAC) only, low MUAC and weight-for-height z-score (WHZ), or low WHZ only) and diet were assessed by linear regression adjusted for age and sex.

Results: Children with MAM had low concentrations of whole-blood PUFAs, particularly n-3 PUFAs. Moreover, children diagnosed with MAM based only on low MUAC had 0.32 (95\% confidence interval $(\mathrm{Cl}), 0.14 ; 0.50)$ and $0.40(95 \% \mathrm{Cl}, 0.16$; 0.63) FA\% lower arachidonic acid (AA) than those recruited based on both low WHZ as well as low MUAC and those recruited with low WHZ only, respectively. Infection and inflammation were associated with low levels of all long-chain (LC)-PUFAs, while hemoglobin was positively associated with whole-blood LC-PUFAs.

Conclusion: While PUFA deficiency was not a general problem, overall whole-blood PUFA concentrations, especially of n-3 PUFAs, were low. Infection, inflammation, hemoglobin, anthropometry and diet were correlates of PUFAs concentrations in children with MAM.

Trial registration: The trial is registered at http://www.isrctn.com (ISRCTN42569496).

Keywords: Essential fatty acids, Undernourished children, Diet, Morbidity

\section{Introduction}

Approximately, 33 million children worldwide suffer from moderate wasting [1]. The current case definition of moderate acute malnutrition (MAM) includes children with moderate wasting, defined as a weight-for-height $\mathrm{z}$-score

\footnotetext{
* Correspondence: yameogocharles69@gmail.com

${ }^{1}$ Centre de Recherche en Sciences Biologiques, Alimentaires et

Nutritionnelles, Université Ouaga I Prof Joseph KI-ZERBO, Ouagadougou, Burkina Faso

${ }^{2}$ Department of Nutrition, Exercise and Sports, University of Copenhagen,

Frederiksberg C, Denmark

Full list of author information is available at the end of the article
}

(WHZ) between -2 and -3 , based on the 2006 WHO growth standard [2], and/or children with a mid-upperarm circumference (MUAC) between 115 and $125 \mathrm{~mm}$ [3]. Children with MAM are at immediate risk of morbidity and mortality from infectious diseases $[4,5]$, and may present with lipid metabolism disturbances likely to impair blood levels of polyunsaturated fatty acids (PUFAs) [6].

Tissue concentrations of PUFAs are reflected in the fatty acid composition of plasma and erythrocytes and the amount of various PUFAs in these pools has been shown to be low in children with severe malnutrition $[7,8]$. Limited data exist on early life intake and blood levels of 
PUFAs in low-income countries among malnourished children [9]. The fatty acid composition in plasma or red blood cells from children with moderate malnutrition has only been investigated in two studies with small samples sizes $[7,10]$. However, in these studies included children with moderate stunting and/or underweight rather than wasting and used different growth references rather than the current WHO growth standards from 2006 [2]. No study was found that investigated fatty acids in children with MAM based on the above definition and compared the fatty acid composition in whole-blood between children diagnosed with MAM based on low MUAC only, low MUAC and WHZ, or low WHZ only.

PUFAs play an important role in early life because they are needed to ensure optimal growth and development of all organ systems [11]. More specifically, linoleic acid (LA), $\alpha$-linolenic acid (ALA), docosahexaenoic acid (DHA) and arachidonic acid (AA) are involved in numerous metabolic processes in addition to acting as a source of energy [12]. LA, incorporated in skin ceramides, is of great importance for skin barrier integrity [13]. DHA is abundant in the cells of the central nervous system and is important for brain development [14]. Furthermore, DHA and AA are incorporated into cell membrane phospholipids and are precursors of lipid mediator signalling molecules $[15,16]$, which play important roles, among others, in the immune system [17]. Considering these important functions, more attention should be paid to PUFA status in children with MAM and it should be investigated how blood PUFA composition correlates with factors such as diet, infections (e.g. diarrhea, malaria), inflammation and anemia and whether there is an association between PUFAs and anthropometry.

Previous studies investigating blood PUFA composition in Burkina Faso, West Africa, have been undertaken in populations not suffering from MAM and in the central region [18-20], an area of the country where groundnuts are widely available and diets are high in fat [21]. Our study was focused on whole-blood PUFA composition among 6-23 months-old children with MAM from the northern region in Burkina Faso, where food insecurity is common. The aim of the study was 1) to describe wholeblood PUFA composition in children with MAM and 2) to identify correlates (e.g. hemoglobin, acute phase proteins, clinical infection, anthropometry and diet) of PUFA composition.

\section{Subjects and methods}

\section{Study setting and participants}

This is a cross-sectional study using baseline data from a prospective nutritional intervention trial among 1609 children with MAM aged 6-23 months. The study took place in the Province du Passoré, Burkina Faso, at five local governmental health centers (Gomponsom,
Latoden, Bagaré, Bokin and Samba). The recruitment area covered a total of 143 villages and a population of approximately 258,000. Recruitment took place from September 2013 to August 2014.

Children, aged 6-23 months, residing in the recruitment area were invited to participate if they were diagnosed with MAM; based on a WHZ between -3 and -2 based on the 2006 WHO growth standard [2] and/or MUAC between $115 \mathrm{~mm}$ and $125 \mathrm{~mm}$ [3]. Children were excluded if they were treated for SAM, if they were already in a nutritional program or if they had been hospitalized within the past 2 months, had medical complications requiring hospitalization, or a severe disability.

\section{Socio-demographic, clinical and diet data collection}

A local nurse carried out a clinical examination and collected data on sociodemographic characteristics as well as 2 week retrospective morbidity. Fever was defined as an axillary temperature $\geq 37.5{ }^{\circ} \mathrm{C}$ [22]. The nurse also collected data on breastfeeding and the childrens' diet using structured questionnaires administered to the caretakers by trained interviewers. With regard to breastfeeding, caretakers were asked whether the child was ever breastfed, currently breastfed and if so how many times the child was breastfed in the previous $24 \mathrm{~h}$. Dietary data was collected using a qualitative 24-h recall interview. More specifically, caretakers were asked what the children consumed in the previous $24-\mathrm{h}$, but no quantities were recorded. If a particular dish was mentioned, caretaker's were asked to list the ingredients. Information recorded during the qualitative 24-h recall interview was then used to complete the food group list section of the questionnaire by answering "yes" or "no" for each food group. The list of 25 food groups was based on internationally available questionnaires from WHO [23], FAO [24] as well as research about diets in Ouagadougou carried out by the Institut de Recherche et Développement [25] and adapted to the local context using dietary information collected during the Treatfood pilot study. For reasons of simplicity, the 25 food groups was then aggregated into eight food groups, namely i) cereals, roots, and tubers; ii) legumes and nuts; iii) liquid oils and fats; iv) dairy; v) flesh foods; vi) eggs; vii) vitamin A-rich fruits and vegetables or viii) other fruits and vegetables as suggested by WHO [23].

\section{Blood sampling and analyses}

During the medical examination up to $2.5 \mathrm{~mL}$ of none fasting venous blood were collected by phlebotomy using needle and syringe. One drop of blood was used to carry out rapid antigen test for Plasmodium falciparum malaria (SD Bioline, Malaria antigen P.f.), and a second drop for determination of hemoglobin level by 
Hemocue (HB 301, Ängelholm, Sweden). A third drop was used to saturate $1 \mathrm{~cm}^{2}$ of a chromatography paper strip treated with $50 \mu \mathrm{g}$ 2,6-di-tert-butyl-4-methylphenol (butylated hydroxytoluene) and $1000 \mu \mathrm{g}$ deferoxamine mesylate salt (both from Sigma-Aldrich, St. Louis, MI, USA) as described previously [26]. The blood spots were allowed to air dry and then the paper strip were kept in cold boxes in the field and stored in zip lock plastic bags at $4{ }^{\circ} \mathrm{C}$ until shipment to Canada. The remaining blood sample was put into a tube with clot activator (BD tube reference \#368492; Becton, Dickinson and Company, Franklin Lakes, USA) and transported in a coldbox at 2$8{ }^{\circ} \mathrm{C}$ to the trial lab, where serum was isolated following centrifugation at 3000 RPM for 5 min (EBA $20 \mathrm{~S}$ Hettich, Tuttlingen, Germany).

Serum was stored at $-20{ }^{\circ} \mathrm{C}$ until shipment to a laboratory in Germany (Vitamin lab, Willstaett, Germany) for analysis. Serum C-reactive protein (CRP) and $\alpha_{1}$-acid glycoprotein (AGP) were determined using a simple sandwich enzyme-linked immunosorbant assay [27]. The thresholds used for defining abnormal values for CRP and AGP were as follows: CRP $>10 \mathrm{mg} / \mathrm{L}$ [28] and AGP $>1 \mathrm{~g} / \mathrm{L}$ [29].

The fatty acid composition of whole-blood was analysed as previously described [30, 31]. In brief, fatty acid methyl esters were prepared from dried blood spots by direct trans-esterification in $14 \%$ boron trifluoride in methanol (Pierce Chemicals, Rockford, IL, USA) with hexane using a convectional block heater set at $95{ }^{\circ} \mathrm{C}$ for $60 \mathrm{~min}$. The organic layer containing the fatty acid methyl esters was collected for analysis on a Varian 3900 gas chromatograph with a CP-8400 autosampler (Varian Inc., Mississauga, ON, Canada) and equipped with a DB-FFAP $15 \mathrm{~m} \times 0.10 \mathrm{~mm}$ i.d. $\times$ $0.10 \mu \mathrm{m}$ film thickness, nitroterephthalic acid modified, polyethylene glycol, capillary column (J\&W Scientific from Agilent Technologies, Mississauga, ON, Canada). Fast gas chromatography settings with hydrogen as the carrier gas were used as previously described [30, 31]. Peaks were identified by retention times through comparison to an external mixed standard sample (GLC-462, Nu Chek Prep Inc., Elysian, MN, USA). Whole-blood fatty acid concentration is given as ( $\mu \mathrm{g}$ fatty acid/100 $\mu \mathrm{L}$ whole-blood) and fatty acid composition data are given as weight percent of individual fatty acids relative to the total fatty acid concentration in each sample (FA\%).

Due to lack of cut-off points for whole-blood Mead acid and n-6 docosapentaenoic acid (DPA), the ratios Mead acid:AA, n-6 DPA:DHA or n-6/n-3 PUFAs were used to define PUFA deficiency. Based on data in healthy well-nourished Danish infants (Lauritzen et al. unpublished data), we considered a Mead acid:AA ratio $>0.02$ as an indicator of PUFA deficiency and a ratio of $n-6$
DPA:DHA $>0.2$ and a $n-6 / n-3$ PUFA ratio $>10.5$ was taken to indicate low n-3 PUFA status.

\section{Anthropometric measures}

Anthropometric measurements were done by trained staff, after standardisation sessions. Weight was measured in duplicate to the nearest $100 \mathrm{~g}$ with an electronic scale (Seca model 881 1021659, Seca GmbH \& Co. KG, Hamburg, Germany) with double weighing function. Length was measured in duplicate to the nearest $1 \mathrm{~mm}$ with a wooden length board. MUAC was measured to the nearest $1 \mathrm{~mm}$ at the midpoint between the olecranon and the acromion process using a standard measuring tape.

\section{Data analysis}

The data were doubled entered into Epidata 3.1 software (Epidata Association, Odense, Denmark). The statistical analysis and tests were performed in Stata 12 (StataCorp, College Station, TX, USA). A WHO WHZ table was used during recruitment at sites, but for data analysis WHZ scores were recalculated using the STATA package "zscore06". Variables were tested for normality using Shapiro-Wilk tests and histograms. Results are shown as mean \pm standard deviation $( \pm \mathrm{SD})$ or median (interquartile range (IQR)). The difference between groups was analyzed by one-way analysis of variance (ANOVA) and p-values from post-hoc pairwise comparisons (three comparisons for each ANOVA test) were Bonferroni adjusted. Multivariable linear regression analysis was performed to assess correlates of individual PUFAs in children with MAM adjusted for age and sex. Statistical significance was set at $p<0.05$.

\section{Results}

Among the 1609 recruited children, a total of 1572 (97.7\%) children had whole-blood fatty acids determined and 37 (2.3\%) did not either due to failure to draw blood or problems during blood analysis at the lab. Upon recruitment, 457 (29\%), 786 (50\%), and 329 (21\%) were diagnosed with MAM based on "MUAC only", "MUAC and WHZ", and "WHZ only", respectively, as previously reported [32]. The median (IQR) age was $11.3(8.2 ; 16)$ months, half $(54.8 \%)$ were girls (Table 1) and $95 \%$ were still breastfed. As reported elsewhere [33], co-morbidity was common. Among the 1572 children with fatty acid data, 593 (38.1\%) children had been ill in the 2 weeks before admission based on maternal recall - hereof 318 (20.2\%) with fever and 277 (17.6\%) with diarrhea. At recruitment, $629(40.2 \%)$ had a positive malaria test, $275(17.5 \%)$ had fever and 83 (5.3\%) had diarrhea. The mean hemoglobin was $10.0( \pm 1.6) \mathrm{g} / \mathrm{dL}$, while the median CRP and AGP were $2.3(0.8 ; 9.5) \mathrm{mg} / \mathrm{L}$ and $1.2(0.9$; 1.6) $\mathrm{g} / \mathrm{L}$, respectively, and $364(23.6 \%)$ had elevated CRP and AGP. 
Table 1 Background data, anthropometric, clinical and biochemical characteristics among 1572 children with moderate acute malnutrition

\begin{tabular}{|c|c|}
\hline Age (months), median (IQR) & $11.3(8.2 ; 16.0)$ \\
\hline $6-11, n(\%)$ & $854(54.3)$ \\
\hline $12-17, n(\%)$ & $447(28.5)$ \\
\hline $18-23, n(\%)$ & $271(17.2)$ \\
\hline \multicolumn{2}{|l|}{ Sex } \\
\hline Boys, n (\%) & $682(45.2)$ \\
\hline Girls, n (\%) & $710(54.8)$ \\
\hline \multicolumn{2}{|l|}{ Breastfeeding status } \\
\hline Still breastfed, n (\%) & $1488(94.8)$ \\
\hline Not breastfed, n (\%) & $82(5.2)$ \\
\hline \multicolumn{2}{|l|}{ Anthropometrics indicators, mean $( \pm S D)$} \\
\hline WHZ & $-2.2( \pm 0.5)$ \\
\hline HAZ & $-1.7( \pm 1.1)$ \\
\hline MUAC (mm) & $122.6( \pm 4)$ \\
\hline \multicolumn{2}{|l|}{ Biochemical } \\
\hline Hemoglobin (g/dL), mean $( \pm \mathrm{SD})$ & $10.0( \pm 1.6)$ \\
\hline AGP (g/L), median (IQR) & $1.2(0.9 ; 1.6)$ \\
\hline CRP (mg/L), median (IQR) & $2.3(0.8 ; 9.5)$ \\
\hline \multicolumn{2}{|l|}{ Morbidity } \\
\hline \multicolumn{2}{|l|}{ III the last two weeks } \\
\hline No, n (\%) & 967 (61.9) \\
\hline Yes, n (\%) & $596(38.1)$ \\
\hline \multicolumn{2}{|l|}{ Diarrhea at recruitment } \\
\hline No, n (\%) & $1489(94.7)$ \\
\hline Yes, n (\%) & $83(5.3)$ \\
\hline \multicolumn{2}{|l|}{ Fever at recruitment } \\
\hline No, n (\%) & $1295(82.5)$ \\
\hline Yes, n (\%) & $275(17.5)$ \\
\hline \multicolumn{2}{|l|}{ Malaria at recruitment } \\
\hline No, n (\%) & $936(59.8)$ \\
\hline Yes, n (\%) & $629(40.2)$ \\
\hline
\end{tabular}

The majority $(85.2 \%)$ of the children had consumed cereals, roots and tubers in the previous $24 \mathrm{~h}$, while 285 (18.1\%), 237 (15.1\%), and 454 (28.9\%) had consumed liquid oils and fats, legumes and nuts or vitamin A-rich fruits and vegetables, respectively (Table 4). More than half $(54.5 \%)$ of the children had also consumed others fruits and vegetables. Almost all children were breastfed (95\%) and only $6(0.4 \%), 60(3.8)$ and $107(6.8 \%)$ had eaten eggs, dairy and flesh foods, respectively, on the day before the examination.
Concentrations and correlates of polyunsaturated fatty acids The mean weight percent of whole-blood LA and ALA were $16.22( \pm 2.26)$ and $0.21( \pm 0.09)$ FA\%, respectively, while that of their respective LC-PUFAs, AA and DHA, were $7.08( \pm 1.53)$ and $1.64( \pm 0.53)$ FA\%, respectively (Table 2). The mean Mead acid:AA ratio of $0.01( \pm 0.00)$ and the generally low mean weight percent of Mead acid $(0.07( \pm 0.03)$ FA\%) indicates that essential fatty acid deficiency was not frequent in the participants. Based on our definition, only $1.21 \%$ of the children with MAM had signs of PUFA deficiency. However, the mean ratio of $0.25( \pm 0.10)$ between $n-6$ DPA and DHA and the mean $n-6 / n-3$ PUFA ratio of $11.23( \pm 2.85)$ indicates low n-3 PUFA status. The percents of children who were above the mean ratio of $0.25( \pm 0.10)$ between $n-6$ DPA and DHA or $11.23( \pm 2.85)$ between $n-6$ and n-3 PUFA were 51.2 and $45.8 \%$ respectively.

Comparisons using ANOVA showed that children recruited based on MUAC only had lower AA than those recruited based on both MUAC and WHZ (6.88 vs. 7.15 FA\%, $p=0.007)$ and WHZ only (6.88 vs. $7.19 \mathrm{FA} \%, p=$ 0.013), but higher ALA than those recruited based on WHZ (0.22 vs. $0.20 \mathrm{FA} \%, p=0.042)$ (Table 3). Furthermore, children recruited based on low MUAC only had lower EPA than those recruited with WHZ only (0.12 vs. $0.14 \mathrm{FA} \%, p=0.040)$. Furthermore, multiple regression

Table 2 Whole blood fatty acids composition in 1572 children with moderate acute malnutrition

\begin{tabular}{|c|c|c|}
\hline & & Mean $( \pm S D)$ \\
\hline Saturated fatty acid & & $45.29( \pm 2.99)$ \\
\hline Monounsaturated fatty acid & & $22.57( \pm 3.39)$ \\
\hline Polyunsaturated fatty acid (PUFA) & & $28.52( \pm 2.78)$ \\
\hline n-6 PUFA & & $25.99( \pm 2.65)$ \\
\hline Linoleic acid (LA) & $18: 2 n-6$ & $16.22( \pm 2.26)$ \\
\hline Dihomo-y-linolenic acid & $20: 3 n-6$ & $0.85( \pm 0.21)$ \\
\hline Arachidonic acid (AA) & $20: 4 n-6$ & $7.08( \pm 1.53)$ \\
\hline Adrenic Acid & $22: 4 n-6$ & $0.95( \pm 0.26)$ \\
\hline n-6 Docosapentaenoic acid (DPA) & $22: 5 n-6$ & $0.38( \pm 0.12)$ \\
\hline n-3 PUFA & & $2.48( \pm 0.65)$ \\
\hline a-Linolenic acid (ALA) & $18: 3 n-3$ & $0.21( \pm 0.09)$ \\
\hline Eicosapentaenoic acid (EPA) ${ }^{a}$ & $20: 5 n-3$ & $0.13(0.10 ; 0.18)$ \\
\hline n-3 Docosapentaenoic acid & $22: 5 n-3$ & $0.43( \pm 0.12)$ \\
\hline Docosahexaenoic acid (DHA) & $22: 6 n-3$ & $1.64( \pm 0.53)$ \\
\hline Mead acid & $20: 3 n-9$ & $0.07( \pm 0.03)$ \\
\hline Mead acid:AA & $20: 3 n-9 / 20: 4 n-6$ & $0.01( \pm 0.00)$ \\
\hline n-6 DPA:DHA & $22: 5 n-6 / 22: 6 n-3$ & $0.25( \pm 0.10)$ \\
\hline n-6 PUFA:n-3 PUFA & & $11.13( \pm 2.85)$ \\
\hline
\end{tabular}


Table 3 Whole blood fatty acids composition by recruitment criteria in 1572 children with moderate acute malnutrition

\begin{tabular}{|c|c|c|c|c|}
\hline & & $\begin{array}{l}\text { MUAC } \\
(n=457)\end{array}$ & $\begin{array}{l}\text { MUAC and WHZ } \\
(n=786)\end{array}$ & $\begin{array}{l}\text { WHZ } \\
(n=329)\end{array}$ \\
\hline & & $\begin{array}{l}\text { Median age (IQR) } \\
9.6(7.3 ; 13.6)\end{array}$ & $\begin{array}{l}\text { Median age (IQR) } \\
11.6(8.4 ; 16.4)\end{array}$ & $\begin{array}{l}\text { Median age (IQR) } \\
13.3(9.9 ; 17.8)\end{array}$ \\
\hline & & Mean FA ( \pm SD) & Mean FA ( \pm SD) & Mean FA ( \pm SD) \\
\hline Saturated fatty acid & & $45.35( \pm 3.38)$ & $45.29( \pm 2.82)$ & $45.24( \pm 2.82)$ \\
\hline Monounsaturated fatty acid & & $22.77( \pm 3.53)$ & $22.57( \pm 3.34)$ & $22.29( \pm 3.31)$ \\
\hline Polyunsaturated fatty acid (PUFA) & & $28.40( \pm 2.69)$ & $28.54( \pm 2.78)$ & $28.68( \pm 2.92)$ \\
\hline n-6 PUFA & & $25.91( \pm 2.53)$ & $25.99( \pm 2.67)$ & $26.11( \pm 2.77)$ \\
\hline Linoleic acid (LA) & $18: 2 n-6$ & $16.37( \pm 2.16)$ & $16.15( \pm 2.31)$ & $16.22( \pm 2.29)$ \\
\hline Dihomo-y-linolenic acid & $20: 3 n-6$ & $0.87( \pm 0.21)$ & $0.85( \pm 0.21)$ & $0.85( \pm 0.21)$ \\
\hline Arachidonic acid (AA) & $20: 4 n-6$ & $6.88( \pm 1.53)^{1,2}$ & $7.15( \pm 1.53)$ & $7.19( \pm 1.54)$ \\
\hline Adrenic Acid & $22: 4 n-6$ & $0.92( \pm 0.26)^{2}$ & $0.96( \pm 0.27)$ & $0.97( \pm 0.27)$ \\
\hline n-6 Docosapentaenoic acid (DPA) & $22: 5 n-6$ & $0.37( \pm 0.13)$ & $0.39( \pm 0.13)$ & $0.38( \pm 0.13)$ \\
\hline n-3 PUFA & & $2.42( \pm 0.64)$ & $2.50( \pm 0.68)$ & $2.51( \pm 0.62)$ \\
\hline a-Linolenic acid (ALA) & $18: 3 n-3$ & $0.22( \pm 0.09)^{2}$ & $0.22( \pm 0.09)$ & $0.20( \pm 0.09)$ \\
\hline Eicosapentaenoic acid (EPA) ${ }^{a}$ & $20: 5 n-3$ & $0.12(0.09 ; 0.18)^{2}$ & $0.13(0.10 ; 0.18)$ & $0.14(0.10 ; 0.20)$ \\
\hline n-3 Docosapentaenoic acid & $22: 5 n-3$ & $0.43( \pm 0.12)$ & $0.45( \pm 0.13)$ & $0.44( \pm 0.12)$ \\
\hline Docosahexaenoic acid (DHA) & $22: 6 n-3$ & $1.60( \pm 0.52)$ & $1.65( \pm 0.54)$ & $1.67( \pm 0.53)$ \\
\hline Mead acid & $20: 3 n-9$ & $0.07( \pm 0.03)$ & $0.07( \pm 0.03)$ & $0.07( \pm 0.02)$ \\
\hline Mead acid:AA & $20: 3 n-9 / 20: 4 n-6$ & $0.01( \pm 0.01)$ & $0.01( \pm 0.00)$ & $0.01( \pm 0.01)$ \\
\hline n-6 DPA:DHA & $22: 5 n-6 / 22: 6 n-3$ & $0.25( \pm 0.10)$ & $0.25( \pm 0.10)$ & $0.24( \pm 0.09)$ \\
\hline n-6 PUFA:n-3 PUFA & & $11.40(2.91)$ & $11.10(2.86)$ & $11.00(2.77)$ \\
\hline
\end{tabular}

Data are given as mean ( \pm standard deviation, SD) weight percent of total whole blood fatty acids, if not otherwise indicated. $p$-value for difference between admission groups are based on ANOVA test with Bonferroni adjustment. Abbreviations: WHZ weigh-for-height z-score, MUAC mid-upper arm circumference ${ }^{1} p<0.05$ between MUAC and, MUAC and WHZ, and ${ }^{2} p<0.05$ between MUAC and WHZ

a Median (interquartile range: $25^{\text {th }} ; 75^{\text {th }}$ percentile) and ANOVA test on log value

analyses, adjusted for age and sex, revealed that the children who were recruited based on MUAC only had 0.32 (95\% confidence interval (CI), 0.14; 0.50) FA\% lower AA than those recruited based on both MUAC and WHZ and 0.40 (95\% CI, 0.16; 0.63) FA\% lower AA than those recruited based on WHZ only. Recruitment based on MUAC only was also associated with 0.09 (95\% CI, 0.01; 0.17) FA\% lower DHA than those recruited based on WHZ only, whereas recruitment based on both MUAC and WHZ was associated with a higher ratio of n-6 DPA relative to DHA (0.01 [95\% CI, 0.00; 0.02]) and higher Mead acid (0.004 [95\% CI, 0.000; 0.007] FA\%) compared to those recruited with WHZ only. Being recruited based on MUAC only compared to WHZ only was positively associated with Mead acid:AA ratio $(\beta=0.001,95 \% \mathrm{CI}$, $0.000 ; 0.002)$. Although median age differed between children recruited based on MUAC only, both MUAC and WHZ, or WHZ only, the difference in AA, DHA and Mead acid:AA across recruitment criteria was not confounded by age, sex and breastfeeding.

Diet was found to be a correlate of whole-blood PUFA status adjusted for age and sex (Table 4). Relative to children who were not breastfed, breastfed children had
1.17 (95\% CI, 0.64; 1.70), 0.75 (95\% CI, 0.39; 1.11), and 0.13 (95\% CI, 0.01; 0.25) FA\% higher LA, AA, and DHA, respectively. Children who had consumed foods from the legumes and nuts group or the oils and fats group in the previous $24 \mathrm{~h}$ had 0.38 (95\% CI, 0.16; 0.59) and 0.32 (95\% CI, 0.12; 0.52) FA\% lower AA, respectively, compared to those who did not consume these foods. This was also the case for children with a dietary intake from the vitamin A-rich fruits and vegetables group. Children with a dietary intake from the oils and fats group or the flesh food group had 0.08 (95\% CI, 0.09; 0.15) and 0.23 (95\% CI, 0.12; 0.33) FA\% higher DHA, respectively compared to those who had not eaten these foods. Dietary intake from the flesh foods group was found to be associated with a higher proportion of DHA relative to that of AA and that was also the case for dietary intake of oils, which were mainly vegetable oils. Dietary intake from both of these food groups was also found to provide a larger difference in the proportion of n-3 PUFAs than in n-6 PUFAs.

Morbidity and biochemical markers of infection were also correlates of whole-blood PUFA content after adjustment for age and sex (Table 5). Children who had 


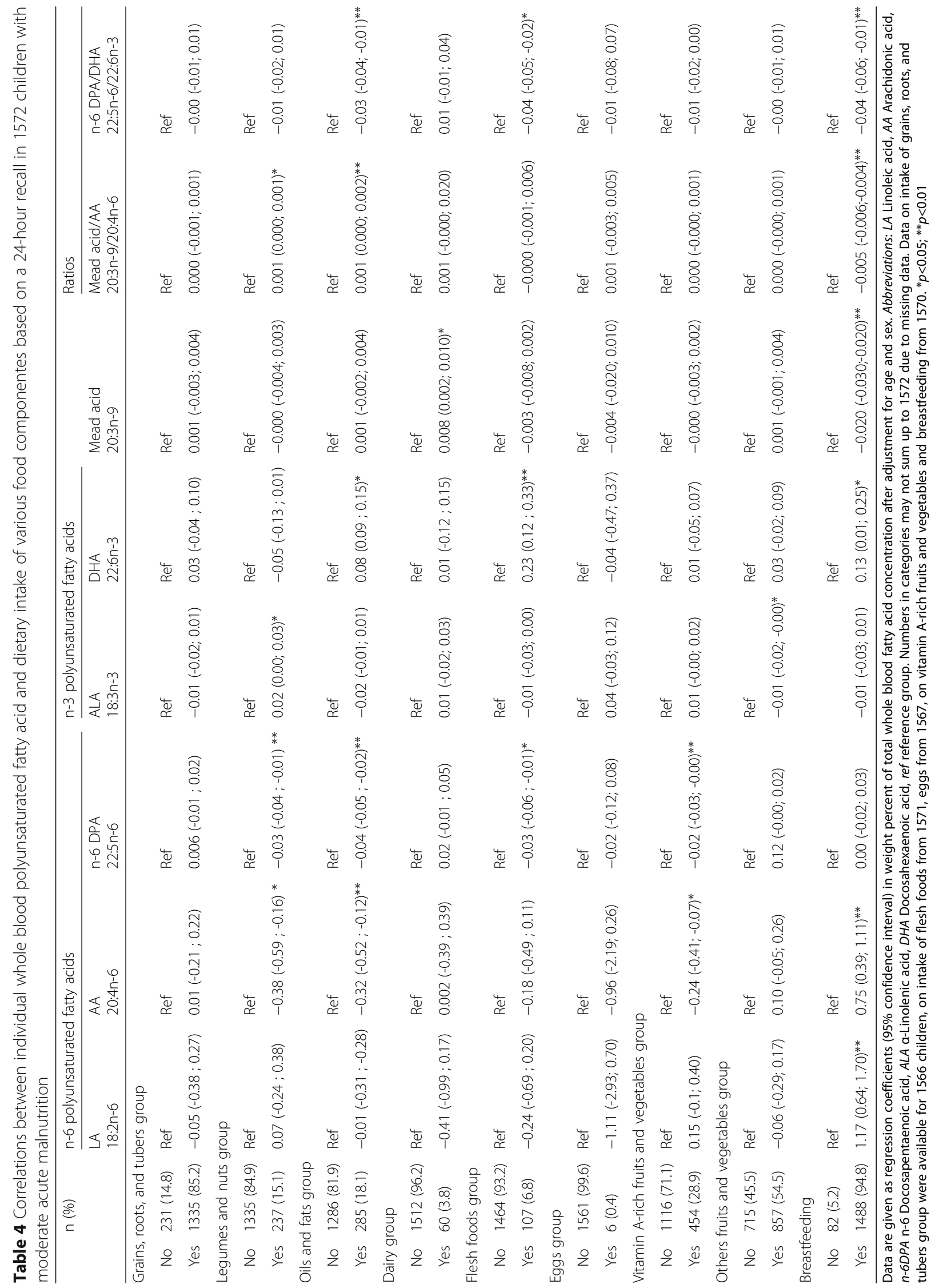




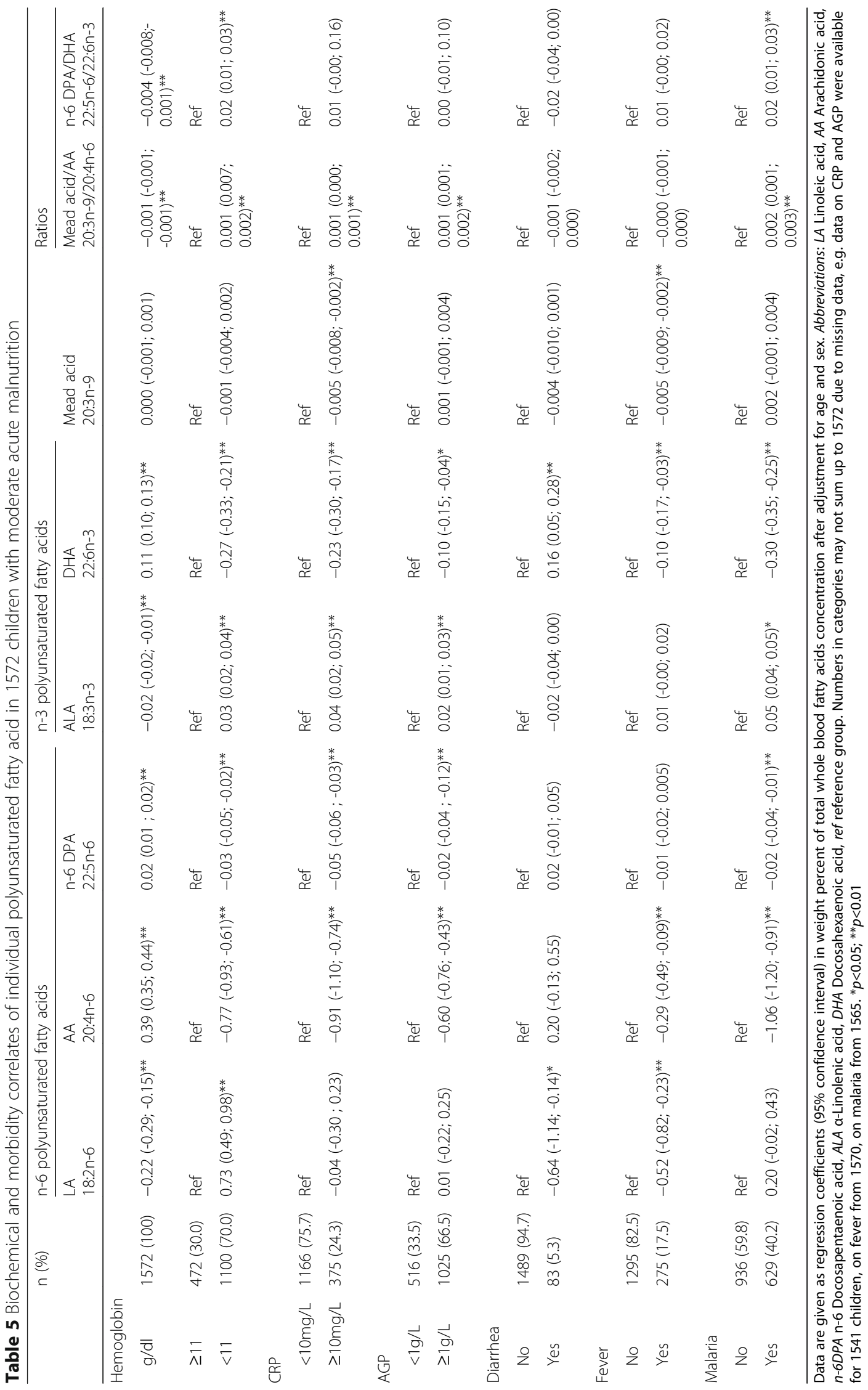


fever at recruitment had lower LA, AA and DHA by 0.52 (95\% CI, 0.23; 0.82), 0.29 (95\% CI, 0.09; 0.49) and 0.10 (95\% CI, 0.03; 0.17) FA\%, respectively. Similarly, fever in the 2 weeks prior to recruitment was associated with low AA and DHA, but not LA. Diarrhea at recruitment was negatively associated with LA, but positively associated with DHA, which was also seen with diarrhea based on maternal recall ( $p<0.05$, data not shown). Children with elevated CRP had 0.91 (95\% CI, 0.74; 1.10 ) and 0.23 (95\% CI, 0.17; 0.30) FA\% lower AA and DHA, respectively, but 0.04 (95\% CI, 0.02; 0.05) FA\% higher ALA. Elevated AGP was also associated with lower AA and DHA (0.60 [95\% CI, 0.43; 0.76] and 0.10 [95\% CI, 0.04; 0.15] FA\%, respectively) but higher ALA (0.02 [95\% CI, 0.01; 0.03] FA\%). A positive malaria test was associated with lower AA and DHA (1.06 [95\% CI, $0.91 ; 1.20$ ] and 0.30 [95\% CI, 0.25; 0.35] FA\%, respectively) and higher ALA (0.05 [95\% CI, 0.04; 0.05] FA\%). Overall, elevated serum CRP and AGP, and a positive malaria test were associated with a higher Mead acid:AA ratio. A positive malaria test was also associated with a higher $n-6$ DPA/DHA ratio. For each $1 \mathrm{~g} / \mathrm{dl}$ increase in hemoglobin, LA and ALA decreased by -0.22 (95\% CI, -0.29; -0.15) and -0.02 (95\% CI, -0.02; -0.01) FA\%, respectively. However, for each $1 \mathrm{~g} / \mathrm{dl}$ increase in hemoglobin, AA and DHA increased by 0.39 (95\% CI, $0.35 ; 0.44)$ and 0.11 (95\% CI, $0.10 ; 0.13) \mathrm{FA} \%$, respectively.

\section{Discussion}

While we found that PUFA deficiency was not a general problem in children with MAM, overall PUFA concentrations, especially of n-3 PUFAs, were low. There could be a number of explanations for this. Firstly, the low levels of whole-blood n-3 PUFAs found in children with MAM could be linked to their diet [34]. The children were from a population with a mainly plant-based diet rich in LA, including peanut oils and peanut basedproducts, cottons seed oils and cereals. Infants and young children in such a population will have an n-3 PUFA intake far below the recommended intake [35]. Previous studies from the central region in Burkina Faso have demonstrated a high proportion of n-6 PUFAs relative to $n-3$ PUFAs in breast milk of healthy mothers nursing 5-months-old infants and this was negatively associated with growth [18]. The low relative levels of $n-3$ PUFAs in children with MAM was compensated for by a high proportion in SFA, which is in line with data from moderately wasted Pakistani children [6]. This high proportion of SFA in our study could be due to more breastfeeding in Burkina Faso as shown in previous studies [18, 36]. Secondly, the low whole-blood n-3 PUFAs could be due to metabolic disturbances occuring with malnutrition [6]. Micronutrient deficiencies such as zinc and iron combined with protein deficiency may impair delta-6 desaturase activity $[5,37]$ and decrease the conversion of ALA to DHA.

As expected, breastfeeding, flesh foods, vegetable oils and fats were found to be correlates of PUFAs in children with MAM. Breast milk is recognised as a good source of LC-PUFAs [38], although this is influenced by maternal diet [39]. Children who were breastfed on the day before blood sampling had higher LA, AA, and DHA compared to those who were not breastfed. Children who consumed flesh foods (meat and fish) in the previous $24 \mathrm{~h}$ also had higher whole-blood $\mathrm{n}-3$ PUFAs and DHA than those who did not consume these types of foods. This is in line with meat and specifically fish being the dominant sources of n-3 LC-PUFAs [34]. A high intake of n-3 LC-PUFAs has been shown to increase EPA and DHA in plasma and tissue phospholipids [40], which is often compensated by a reduction in LA, AA and n-6 DPA.

With regard to anthropometry, children recruited by MUAC only seem to have lower whole-blood n-6 PUFAs and n-3 PUFAs compared to those recruited by WHZ only. Specifically, recruitment by MUAC only was associated with low AA and DHA and this could be related to a low muscle mass. Low MUAC only has been shown to be related to low arm muscle mass [41], which is generally associated with hypercortisolemia occuring with malnutrition [42]. Hypercortisolemia increases resting energy expenditure fueled by increased oxidation of fat [43], and this may decrease whole-blood LC-PUFA status in children with low MUAC. Another explanation could be that intake of LC-PUFAs and protein quality could be related, as seen in a Canadian population [44], and thus affect muscle and whole-blood LC-PUFAs in parallel. The tissue most affected by malnutrition in the first months of life is muscle mass [45]. It could also be speculated that low MUAC is more related to long-term inflammation, which may be the cause of low PUFA status.

Markers of infection and inflammation were negatively associated with whole-blood PUFAs. This finding could be explained by an infection-induced supression of appetite and could be a direct effect of dietary intake, but may also be related to impaired absorption, poor nutrient utilization and/or increased nutrient catabolism caused by the inflammatory state $[46,47]$. We speculate that the negative association of LA but positive association of DHA with diarrhea could be due to low absorption of fat during diarrhea resulting in low plasma triacylglycerols and thus, relatively higher levels of DHA from the blood cells. Fever during infection leads to an increase in energy requirement [48] and possibly an increased use of PUFAs as fuel. Infection-induced inflammation has been suggested to increase LC-PUFA catabolism, 
mainly of AA and DHA for the production of proand anti-inflammatory mediators [49] and is likely contributing to the reduced AA and DHA seen in the MAM children, specifically those with low MUAC. Finally, hemoglobin was found to positively correlate with whole-blood LC-PUFAs, mostly AA and DHA, which could possibly be due to their incorporation in phospholipids of erythrocyte membranes [14]. The negative association between hemoglobin and wholeblood LA could be caused by a preferential inorporation of LC-PUFAs in erythrocyte membranes at the expense of LA. The association with anemia and hemoglobin may therefore in part reflect changes in blood composition (erythrocyte number) and may therefore not be an ideal indicator of whole blood PUFA status.

The current study is the first to investigate wholeblood PUFA composition and correlates among a large sample of children with MAM. Also, this is the first study to show a relationship between low MUAC and PUFAs. The diversity of correlates such as infection, inflammation, diet, and anthropometry highlight the complexity of factors which may affect whole-blood PUFA composition in children with MAM, and these factors should be taken into account in studies investigating PUFAs among malnourished children. However, the study had some limitations, in particular the lack of a control group from the same study setting, which makes it difficult to differentiate between potential effects of diet and the overall low nutritional status. Differences in diet could confound differences in nutritional status even if we had been able to collect data from a wellnourished group of children in Burkina Faso. The diet information was based on a single qualitative 24-h recall which may have reduced accuracy and thus, reduced the association between diet and PUFAs. The observed associations were however meaningful. Furthermore, PUFA status was only based on a single non-fasting wholeblood sample and may not provide a measure of long-term status as is the case for example in adipose tissue samples [50], but whole-blood EPA and DHA correlates with and predicts erythrocyte EPA and DHA [51]. It may however, match the short-term collection of dietary information and also morbidity, which was based only on recall data from the previous 2 weeks and data from clinical examinations on the day of recruitment.

In conclusion, although PUFA deficiency based on the definition used here was not common, children with MAM in Burkina Faso have low whole-blood n-3 PUFA concentrations. Low n-3 PUFA levels are likely to be, to some extent, explained by a low intake of animal based foods, vegetable oils and fat sources of n-3 PUFA, or by other correlating factors such anemia, infection and inflammation. Children with low MUAC seem to have lower whole-blood PUFAs compared to children with MAM based on WHZ. This observation needs to be confirmed in future investigations among children with MAM.

\section{Abbreviations \\ AA: Arachidonic acid; AGP: $a_{1}$-acid glycoprotein; ALA: a-linolenic acid; ANOVA: Analysis of variance; Cl: Confidence interval; CRP: C-reactive protein; DHA: Docosahexaenoic acid; DPA: Docosapentaenoic acid; EPA: Eicosapentaenoic acid; FA\%: Fatty acid percent; IQR: Interquartile range; LA: Linoleic acid; LC-PUFA: Long-chain polyunsaturated fatty acid; MAM: Moderate acute malnutrition; MUAC: Mid-upper-arm-circumference; MUFA: Monounsaturated fatty acid; PUFA: Polyunsaturated fatty acid; SAM: Severe acute malnutrition; SD: Standard deviation; SFA: Saturated fatty acid; WHO: World health organization; WHZ: Weight-for-height z-score}

\section{Acknowledgements}

We are grateful to the study participants and their families, and the staff of the Alliance for International Medical Action (ALIMA) for their valuable contribution to this study. We thank the Ministry of Health in Burkina Faso, the health and village authorities in Province du Passoré, and the staff at the health centers and for their support to this study.

\section{Funding}

The study was funded by the Danish International Development Assistance (DANIDA, 09-097 LIFE), Médecins Sans Frontières (MSF Denmark \& MSF Norway), World Food Program (WFP), which was part of a donation to WFP from a generous support of the American people through the support of the U.S. Agency for International Development's Office of Food for Peace (USAID). Alliance for International Medical Action (ALIMA) and the European Union's humanitarian aid funds, in partnership with Action Contre la Faim (ACF), Arvid Nilsson foundation.

The funding agencies had no role in the design of study, data collection and analysis, or presentation of the results.

\section{Availability of data and materials}

All data generated or analysed during this study are included in this published article.

\section{Authors' contributions}

The authors' contributions to the manuscript were as follows: MJHR, AB, SS, VBC, KFM, and HF conceived and designed the study; CWY, BC, CF, MJHR and KDS conducted the research; CWY, DFJ, HF and LL analyzed the data and $C W Y$ wrote the first draft of the manuscript and had primary responsibility for the final content. CWY, BC, CF, MJHR, DFJ, KDS, AB, SS, AST, VBC, KFM, HF and $L L$ made revisions to the manuscript and read and approved the final submitted version of the manuscript.

\section{Ethics approval and consent to participate}

The study protocol was approved by the Ethics Committee for Health Research in Burkina Faso (2012-8-059) and consultative approval was obtained from the Danish National Committee on Biomedical Research Ethics (1208204). Consent was obtained verbally and in writing (signature or fingerprints) from caretakers of the children before inclusion. The information and consent form was translated into the local language and back translated to ensure accuracy. The study was carried out in accordance with the declaration of Helsinki and international ethical guidelines for biomedical research involving human subjects, published by the Council for International Organizations of Medical Sciences. Medical treatment was provided according to an adapted version of the Integrated Management of Childhood IIIness guidelines and national protocol. This study was a part of Treatfood trial which was registered at http://www.isrctn.com (ISRCTN42569496).

\section{Consent for publication}

"Not applicable".

Competing interests

The authors declare that they have no competing interests. 


\section{Publisher's Note}

Springer Nature remains neutral with regard to jurisdictional claims in published maps and institutional affiliations.

\section{Author details \\ ${ }^{1}$ Centre de Recherche en Sciences Biologiques, Alimentaires et} Nutritionnelles, Université Ouaga I Prof Joseph KI-ZERBO, Ouagadougou, Burkina Faso. ${ }^{2}$ Department of Nutrition, Exercise and Sports, University of Copenhagen, Frederiksberg C, Denmark. ${ }^{3}$ Département Biomédical et Santé Publique, Institut de Recherche en Sciences de la Santé, 03 BP 7192 Ouagadougou 03, Burkina Faso. ${ }^{4}$ Department of Infectious Diseases, Rigshospitalet, Copenhagen, Denmark. ${ }^{5}$ Department of Kinesiology, University of Waterloo, Waterloo, ON, Canada. ${ }^{6}$ Tampere Centre for Child Health Research, 33014 University of Tampere, Tampere, Finland. ${ }^{7}$ Alliance for International Medical Action, Dakar, Sénégal. ${ }^{8}$ Médecins Sans Frontières Denmark, Copenhagen, Denmark.

Received: 10 January 2017 Accepted: 25 June 2017

Published online: 13 July 2017

\section{References}

1. Black RE, Victora CG, Walker SP, et al. Maternal and child undernutrition and overweight in low-income and middle-income countries. Lancet. 2013;382:427-51.

2. WHO | WHO Child Growth Standards (2006): Length/height-for-age, weight-forage, weight-for-length, weight-for-height and body mass index-for-age: methods and development. WHO press World Health Organization 1211 Geneva 27.

3. UNCHR. Guidelines for selective feeding: the management of malnutrition in emergencies. Publique health and HIV section, Geneva. 2011. Internet: http://www.unhcr.org/4b7421fd20.pdf. Accessed 15 Apr 2016.

4. Black RE, Allen LH, Bhutta ZA, et al. Maternal and child undernutrition: global and regional exposures and health consequences. Lancet. 2008;371:243-60.

5. Olofin I, McDonald CM, Ezzati M, et al. Nutrition impact model study (anthropometry cohort pooling). associations of suboptimal growth with allcause and cause-specific mortality in children under five years: a pooled analysis of ten prospective studies. PLoS One. 2013;8:e64636.

6. Smit EN, Dijkstra JM, Schnater TA, et al. Effects of malnutrition on the erythrocyte fatty acid composition and plasma vitamin E levels of Pakistani children. Acta Paediatr. 1997:86:690-5.

7. Koletzko B, Abiodun PO, Larya MD, et al. Fatty acid composition of plasma lipids in Nigerian children with protein-energy malnutrition. Eur J Pediatr. 1986;145:109-15.

8. Marin MC, De Tomas ME, Mercurie O, et al. Interrelationship between protein-energy malnutrition and essential fatty acid deficiency in nursing children. Am J Clin Nutr. 1991;53:466-8.

9. Briend A, Dewey KG, Reinhardt GA. Fatty acid status in early life in lowincome countries- overview of the situation, policy and research priorities. Matern Child Nutr. 2011;7:141-8.

10. Squali Houssaïni FZ, Foulon T, Payen N, et al. Plasma fatty acid status in Moroccan children: increased lipid peroxidation and impaired polyunsaturated fatty acid metabolism in protein-calorie malnutrition. Biomed Pharmacother. 2001;55:155-62.

11. Hornstra G. Essentials fatty acids in mothers and their neonates. Am J Clin Nutr. 2000;71:1262S-9S.

12. Prentice AM, Paul AA. Fat and energy needs of children in developing countries. Am J Clin Nutr. 2000;72:1253S-65S.

13. Hansen HS, Jensen B. Essential function of linoleic acid esterified in acylglucosylceramide and acylceramide in maintaining the epidermal water permeability barrier. Evidence from feeding studies with oleate, linoleate, arachidonate, columbinate and alpha-linolenate. Biochim Biophys Acta. 1985:834:357-63.

14. Michaelsen KF, Lauritzen L, Mortensen EL. Effects of Breast-feeding on cognitive function. In: GR Boldberg, Prentice A, Filteau S, Simondon K (Eds.). Adv Exp Med Biol. 2009;639:199-215.

15. Innis SM. Essential fatty acids in growth and development. Prog Lipid Res. 1991;30:39-103.

16. Simopoulos AP. Omega-3 fatty acids in health and diseases and in growth and development. Am J Clin Nutr. 1991;54:438-63.

17. Ganapathy S. Long chain polyunsaturated fatty acid and immunity in infants. India Pediatrics. 2009:46:785-90.
18. Rocquelin G, Kiffer J, Tapsoba S, et al. High proportions of n-6 polyunsaturated fatty acids in mature milk of mothers in Ouagadougou, Burkina Faso. Acta Pediatr. 2001;90:450-2.

19. Thiombiano-Coulibaly N, Rocquelin G, Eymard-Duvernay S, et al. Seasonal and environmental effects on breast milk fatty acids in Burkina Faso and the need to improve the omega 3 PUFA content. Acta Pediatr. 2003:92:1388-93.

20. Rocquelin G, Tapsoba S, Kiffer J, et al. Human milk fatty acids and growth of infants in Brazzaville (The Congo) and Ouagadougou (Burkina Faso). Pub Health Nutr. 2003;6:241-7.

21. Ministère de l'Agriculture, de l'Hydraulique Et des Ressources Halieutiques. Enquête nationale sur I'Insécurité alimentaire et la malnutrition. In: Rapport définitif. 2009. p. 60-80.

22. Ministère de la Santé. Prise en charge intégrée des maladies de l'enfant (PCIME). Plan de couverture 2005-2010. Rapport 2010. 42p.

23. World Health Organisation, AED, USAID, FANTA, University of California Davis, IFPRI, UNICEF. Indicators for assessing infant and young child feeding indicators. Part II. Geneva: WHO Press; 2010.

24. Food an Agricultural Organisation (FAO), European Union. Guidelines for measuring household and individual dietary diversity. FAO, Viale delle Terme di Caracalla, 00153 Rome, Italy; 2011.

25. Danel P. Etude de la Consommation Alimentaire à Ouagadougou, Burkina Faso. Typologie des régimes. IRD de Ouagadougou 688, avenue Professeur Joseph Ki-Zerbo Ouagadougou - secteur 4; 2005.

26. Metherel AH, Stark KD. Cryopreservation prevents iron-initiated highly unsaturated fatty acid loss during storage of human blood on chromatography paper at $220^{\circ} \mathrm{C}$. J Nutr. 2015;145:654-60.

27. Erhardt J, Estes J, Pfeiffer $C_{\text {, }}$ et al. Combined measurement of ferritin, soluble transferrin receptor, retinol binding protein, and C-reactive protein by an inexpensive, sensitive, and simple sandwich enzyme-linked immunosorbent assay technique. J Nutr. 2004;134:3127-32.

28. Kushner I. Acute phase reactants. In: UpToDate [Internet]. Waltam, MA; 2015 Available from: www.uptodate.com. Accessed 10 Nov 2015.

29. Raiten D, Sakr Ashour F, Ross A, et al. Inflammation and Nutritional Science for Programs/Policies and Interpretation of Research Evidence (INSPIRE). J Nutr. 2015. doi: 10.3945/jn.114.194571

30. Metherel AH, Aristizabal Henao JJ, Stark KD. EPA and DHA levels in whole blood decrease more rapidly when stored at $-20^{\circ} \mathrm{C}$ as compared with room temperature, 4 and $-75^{\circ} \mathrm{C}$. Lipids. 2013:48:1079-91. doi:10.1007/s11745-013-3827-x.

31. Metherel AH, Hogg RC, Buzikievich LM, et al. Butylated hydroxytoluene can protect polyunsaturated fatty acids in dried blood spots from degradation for up to 8 weeks at room temperature. Lipids Health Dis. 2013;12:22. doi:10.1186/1476-511X-12-22.

32. Fabiansen $\mathrm{C}$, Phelan KP, Cichon B, et al. Short children with a low midupper arm circumference respond to food supplementation: an observational study from Burkina Faso. Am J Clin Nutr. 2016;103:415-21.

33. Cichon B, Fabiansen C, Yaméogo CW et al. Children with moderate acute malnutrition have inflammation not explained by maternal reports of illness and clinical symptoms: a cross-sectional study in Burkina Faso. BMC Nutr [Internet]. BMC Nutrition; 2016; 2:57. Available from: http://bmcnutr. biomedcentral.com/articles/10.1186/s40795-016-0096-0

34. Smit EN, Muskiet FAJ, Boersma ER. The possible role of essential fatty acids in the pathophysiology of malnutrition: a review. Prostaglandins Leukot Essent Fatty Acids. 2004:71:241-50.

35. Michaelsen KF, Dewey KG, Perez-Exposito AB, et al. Food sources and intake of n-6 and n-3 fatty acids in low-income countries with emphasis on infants, young children (6-24 months), and pregnant and lactating women. Matern Child Nutr. 2011;7:124-40.

36. Rocquelin G, Tapsoba S, Dop MC, et al. Lipid content and essential fatty acid (EFA) composition of mature Congolese breast milk are influenced by mothers'nutritional status: impact on infants' EFA supply. Eur J Clin Nutr. 1998:52:164-71.

37. Smuts CM, Tichelaar HY, van Jaarsveld PJ, et al. The effect of iron fortification on the fatty acid composition of plasma and erythrocyte membranes in primary school children with and without iron deficiency. Prostaglandins Leukot Essent Fatty Acids. 1995;52:59-67.

38. Brenna JT, Varamini B, Jensen RG, et al. Docosahexaenoic and arachidonic acid concentrations in human breast milk worldwide. Am J Clin Nutr. 2007; 85:1457-64.

39. Innis SM. Impact of maternal diet on human milk composition and neurological development of infants. Am J Clin Nutr. 2014:99:734S-41S. doi:10.3945/ajen.113.072595. 
40. Lloyd JF, Ekhard EZ. Present knowledge in nutrition. 7th ed. Washington, D. C.: ILSI Press, International Life Sciences Institute; 1996. p. 58-66.

41. Trowbridge $F L$, Hiner $C D$, Robertson AD. Arm muscle indicators and creatinine excretion in children. Am J Clin Nutr. 1982:36:691-6.

42. Rao JKS, Srikantia SG, Gopalan C. Plasma cortisol levels in protein-calorie malnutrition. Arch Dis Child. 1968:43:365-7.

43. Brillon DJ, Zheng B, Campbell RG, et al. Effect of cortisol on energy expenditure and amino acid metabolism in humans. Am J Physiol. 1995;268: 501-13.

44. Stark KD, Patterson AC. EPA and DHA-protein, not fat is "where it's at"? Prostaglandins Leukot Essent Fatty Acids. 2012;87:49-51. doi:10.1016/.jplefa. 2012.05.002

45. McFie J, Welbourn HF. Effect of malnutrition in infancy on the development of bone, muscle and fat. J Nutr. 1962;76:97-105.

46. Rodríguez L, Cervantes E, Ortiz R. Malnutrition and gastrointestinal and respiratory infections in children: a public health problem. Int J Environ Res Publ Health. 2011:8:1174-205. doi:10.3390/ijerph8041174.

47. Tomkins A, Watson F. Malnutrition and Infection - A Review - Nutrition Policy Discussion Paper No. 5. United Nations - Administrative Commitee on Coordination - Subcommitee on Nutrition; 1989:3-30.

48. Katona P, Katona-Apte J. The interaction between nutrition and infection. Clin Infect Dis. 2008:46:1582-8.

49. Calder PC. Omega-3 fatty acids and inflammatory processes. Nutrients. 2010; 2:355-74. doi:10.3390/nu2030355.

50. Baylin A, Campos H. The use of fatty acid biomarkers to reflect dietary intake. Curr Opin Lipidol. 2006:17:22-7.

51. Stark KD, Aristizabal Henao JJ, Metherel AH, et al. Translating plasma and whole blood fatty acid compositional data into the sum of eicosapentaenoic and docosahexaenoic acid in erythrocytes. Prostaglandins Leukot Essent Fatty Acids. 2016;104:1-10. doi:10.1016/j.plefa.2015.11.002.

\section{Submit your next manuscript to BioMed Central and we will help you at every step:}

- We accept pre-submission inquiries

- Our selector tool helps you to find the most relevant journal

- We provide round the clock customer support

- Convenient online submission

- Thorough peer review

- Inclusion in PubMed and all major indexing services

- Maximum visibility for your research

Submit your manuscript at www.biomedcentral.com/submit

) Biomed Central 